\title{
Dealing with inconsistencies in the representation of ordinal information by a 2-additive Choquet integral
}

\author{
Brice Mayag $^{1}$ Michel Grabisch $^{2}$ Christophe Labreuche ${ }^{3}$
}

\author{
${ }^{1}$ École Centrale Paris, Grande Voie des Vignes, F-92295 Châtenay-Malabry Cedex, France, brice.mayag@ecp.fr \\ ${ }^{2}$ University of Paris I, C.E.S, 106-112 Boulevard de l'Hôpital, 75013 Paris, France, michel.grabisch@univ-paris1.fr \\ ${ }^{3}$ Thales R \& T, 1 avenue Augustin Fresnel, 91767 Palaiseau cedex, France, christophe.labreuche@thalesgroup.com
}

\begin{abstract}
We propose an algorithm to solve inconsistencies when the preferences of a decision-maker, given by a strict and an indifference relations on a set of binary actions, are not representable by a 2 -additive Choquet integral. According to the characterization of this type of information, these inconsistencies arise from the violation of the MOPI property or occur in the presence of a strict cycle in his preferences.
\end{abstract}

Keywords: MCDA; Choquet integral; 2-additive capacity; MOPI Property

\section{Introduction}

Multiple Criteria Decision Aid (MCDA) aims at helping a decision maker (DM) in the representation of his preferences over a set of alternatives, on the basis of several criteria which are often contradictory. One possible model is the transitive decomposable one where an overall utility is determined for each option. In this category, we have the model based on Choquet integral, especially the 2additive Choquet integral (Choquet integral w.r.t. a 2 -additive) $[6,8,14]$. The 2 -additive Choquet integral is defined w.r.t. a capacity (or nonadditive monotonic measure, or fuzzy measure), and can be viewed as a generalization of the arithmetic mean. Any interaction between two criteria can be represented and interpreted by a Choquet integral w.r.t. a 2-additive capacity, but not more complex interaction.

Usually the DM is supposed to be able to express his preference over the set of all alternatives $X$. Because this is not feasible in most of practical situations (the cardinality of $X$ may be very large), the $\mathrm{DM}$ is asked to give, using pairwise comparisons, an ordinal information (a preferential information containing only a strict preference and an indifference relations) on a subset $X^{\prime} \subseteq X$, called reference set. The set $X^{\prime}$ we use in this paper is the set of binary alternatives or binary actions denoted by $\mathcal{B}$. A binary action is an (fictitious) alternative representing a prototypical situation where on a given subset of at most two criteria, the attributes reach a satisfactory level $\mathbf{1}$, while on the remaining ones, they are at a neutral level (neither satisfactory nor unsatisfactory) 0.

We are interested in the following problem: how to help the DM when the representation of his preference by a 2-additive Choquet integral lead to inconsistencies? We propose here an original algorithm to manage these inconsistencies. This algorithm is based on the characterization theorem of the representation of an ordinal information by a 2-additive Choquet integral [13]. Therefore the recommendations we suggest to the DM are related to the definition of the MOPI property, the main condition to satisfy in this representation.

After some basic notions given in the next section, we present in Section 3 the recommendations we suggest to the DM in order to have consistent judgements. Finally, we end by the presentation of our algorithm.

\section{Basic concepts}

Let us denote by $N=\{1, \ldots, n\}$ a finite set of $n$ criteria and $X=X_{1} \times \cdots \times X_{n}$ the set of actions (also called alternatives or options), where $X_{1}, \ldots, X_{n}$ represent the point of view or attributes. For all $i \in N$, the function $u_{i}: X_{i} \rightarrow \mathbb{R}$ is called a utility function. Given an element $x=\left(x_{1}, \ldots, x_{n}\right)$, we set $U(x)=\left(u_{1}\left(x_{1}\right), \ldots, u_{n}\left(x_{n}\right)\right)$. For a subset $A$ of $N$ and actions $x$ and $y$, the notation $z=\left(x_{A}, y_{N-A}\right)$ means that $z$ is defined by $z_{i}=x_{i}$ if $i \in A$, and $z_{i}=y_{i}$ otherwise.

\subsection{Choquet integral w.r.t. a 2-additive capacity}

The Choquet integral w.r.t. a 2-additive capacity [6], called for short a 2-additive Choquet integral, is a particular case of the Choquet integral [8, 9, 14]. This integral generalizes the arithmetic mean and takes into account interactions between criteria. A 2-additive Choquet integral is based on a 2-additive capacity [4, 8] defined below and its Möbius transform $[3,7]$ :

\section{Definition 1.}


1. A capacity on $N$ is a set function $\mu: 2^{N} \rightarrow$ $[0,1]$ such that:

(a) $\mu(\emptyset)=0$

(b) $\mu(N)=1$

(c) $\forall A, B \in 2^{N},[A \subseteq B \Rightarrow \mu(A) \leq \mu(B)]$ (monotonicity).

2. The Möbius transform [3] of a capacity $\mu$ on $N$ is a function $m: 2^{N} \rightarrow \mathbb{R}$ defined by:

$$
m(T):=\sum_{K \subseteq T}(-1)^{|T \backslash K|} \mu(K), \forall T \in 2^{N} .
$$

When $m$ is given, it is possible to recover the original $\mu$ by the following expression:

$$
\mu(T):=\sum_{K \subseteq T} m(K), \forall T \in 2^{N} .
$$

For a capacity $\mu$ and its Möbius transform $m$, we use the following shorthand: $\mu_{i}:=\mu(\{i\}), \mu_{i j}:=$ $\mu(\{i, j\}), m_{i}:=m(\{i\}), m_{i j}:=m(\{i, j\})$, for all $i, j \in N, i \neq j$. Whenever we use $i$ and $j$ together, it always means that they are different.

Definition 2. A capacity $\mu$ on $N$ is said to be 2additive if

- For all subsets $T$ of $N$ such that $|T|>2$, $m(T)=0$;

- There exists a subset $B$ of $N$ such that $|B|=2$ and $m(B) \neq 0$.

The following important Lemma shows that a 2additive capacity is entirely determined by the value of the capacity on the singletons $\{i\}$ and pairs $\{i, j\}$ of $2^{N}$ :

\section{Lemma 1.}

1. Let $\mu$ be a 2-additive capacity on $N$. We have for all $K \subseteq N,|K| \geq 2$,

$$
\mu(K)=\sum_{\{i, j\} \subseteq K} \mu_{i j}-(|K|-2) \sum_{i \in K} \mu_{i} .
$$

2. If the coefficients $\mu_{i}$ and $\mu_{i j}$ are given for all $i, j \in N$, then the necessary and sufficient conditions that $\mu$ is a 2-additive capacity are:

$$
\begin{gathered}
\sum_{\{i, j\} \subseteq N} \mu_{i j}-(n-2) \sum_{i \in N} \mu_{i}=1 \\
\mu_{i} \geq 0, \forall i \in N
\end{gathered}
$$

For all $A \subseteq N,|A| \geq 2, \forall k \in A$,

$$
\sum_{i \in A \backslash\{k\}}\left(\mu_{i k}-\mu_{i}\right) \geq(|A|-2) \mu_{k} .
$$

Proof. See [6].
For an alternative $x:=\left(x_{1}, \ldots, x_{n}\right) \in X$, the expression of the Choquet integral w.r.t. a capacity $\mu$ is given by:

$$
\begin{aligned}
& C_{\mu}(U(x)):=\sum_{i=1}^{n}\left(u_{\tau(i)}\left(x_{\tau(i)}\right)-u_{\tau(i-1)}\left(x_{\tau(i-1)}\right)\right) \\
& \mu(\{\tau(i), \ldots, \tau(n)\}) \\
& \text { where } \tau \text { is a permutation on } N \text { such } \\
& \text { that } u_{\tau(1)}\left(x_{\tau(1)}\right) \leq u_{\tau(2)}\left(x_{\tau(2)}\right) \leq \cdots \quad \text { a } \\
& u_{\tau(n-1)}\left(x_{\tau(n-1)}\right) \leq u_{\tau(n)}\left(x_{\tau(n)}\right), \quad \text { and } \\
& u_{\tau(0)}\left(x_{\tau(0)}\right):=0 .
\end{aligned}
$$

The 2-additive Choquet integral can be written also as follows [9]:

$C_{\mu}(U(x))=\sum_{i=1}^{n} v_{i} u_{i}\left(x_{i}\right)-\frac{1}{2} \sum_{\{i, j\} \subseteq N} I_{i j}\left|u_{i}\left(x_{i}\right)-u_{j}\left(x_{j}\right)\right|$

where $v_{i}=\sum_{K \subseteq N \backslash i} \frac{(n-|K|-1) !|K| !}{n !}(\mu(K \cup i)-$ $\mu(K))$ is the importance of criterion $i$ corresponding to the Shapley value of $\mu$ [17] and $I_{i j}=\mu_{i j}-\mu_{i}-\mu_{j}$ is the interaction index between the two criteria $i$ and $j[6,15]$.

\subsection{Binary actions and relations}

MCDA methods based on multiattribute utility theory, e.g, UTA [19], robust methods $[1,5,11]$, require in practice a preferential information of the DM on a subset $X_{R}$ of $X$ because of the cardinality of $X$ which can be very large. The set $X_{R}$ is called reference subset and it is generally chosen by the DM. His choice may be guided by his knowledge about the problem addressed, his experience or his sensitivity to one or more particular alternatives, etc. This task is often difficult for the DM, especially when the alternatives are not known in advance, and sometimes his preferences on $X_{R}$ are not sufficient to specify all the parameters of the model as interaction between criteria. For instance, in the problem of the design of a complex system for the protection of a strategic site [16], it is not easy for the DM to choose $X_{R}$ himself because these systems are not known a priori. For these reasons, we suggest him to use as a reference subset a set of fictitious alternatives called binary actions defined below. We assume that the DM is able to identify for each criterion $i$ two reference levels:

1. A reference level $\mathbf{1}_{i}$ in $X_{i}$ which he considers as good and completely satisfying if he could obtain it on criterion $i$, even if more attractive elements could exist. This special element corresponds to the satisficing level in the theory of bounded rationality of Simon [18].

2. A reference level $\mathbf{0}_{i}$ in $X_{i}$ which he considers neutral on $i$. The neutral level is the absence of attractiveness and repulsiveness. The existence of this neutral level has roots in psychology [20], and is used in bipolar models [21]. 
We set for convenience $u_{i}\left(\mathbf{1}_{i}\right)=1$ and $u_{i}\left(\mathbf{0}_{i}\right)=0$. Because the use of Choquet integral requires to ensure the commensurateness between criteria, the previous reference levels can be used in order to define the same scale on each criterion [10, 12]. More details about these reference levels can be found in $[8,9]$.

We call a binary action or binary alternative, an element of the set

$\mathcal{B}=\left\{\mathbf{0}_{N},\left(\mathbf{1}_{i}, \mathbf{0}_{N-i}\right),\left(\mathbf{1}_{i j}, \mathbf{0}_{N-i j}\right), i, j \in N, i \neq j\right\} \subseteq X$

where

- $\mathbf{0}_{N}=\left(\mathbf{1}_{\emptyset}, \mathbf{0}_{N}\right)=: a_{0}$ is an action considered neutral on all criteria.

- $\left(\mathbf{1}_{i}, \mathbf{0}_{N-i}\right)=: a_{i}$ is an action considered satisfactory on criterion $i$ and neutral on the other criteria

- $\left(\mathbf{1}_{i j}, \mathbf{0}_{N-i j}\right)=: a_{i j}$ is an action considered satisfactory on criteria $i$ and $j$ and neutral on the other criteria.

Using the Choquet integral, we get the following consequences:

1. For any capacity $\mu$,

$$
C_{\mu}\left(U\left(\left(\mathbf{1}_{A}, \mathbf{0}_{N-A}\right)\right)\right)=\mu(A), \forall A \subseteq N .
$$

2. Using Equation (2), we have for any 2-additive capacity $\mu$ :

$$
\begin{gathered}
C_{\mu}\left(U\left(a_{0}\right)\right)=0 \\
C_{\mu}\left(U\left(a_{i}\right)\right)=\mu_{i}=v_{i}-\frac{1}{2} \sum_{k \in N, k \neq i} I_{i k} \\
C_{\mu}\left(U\left(a_{i j}\right)\right)=\mu_{i j}=v_{i}+v_{j}-\frac{1}{2} \sum_{k \in N, k \notin\{i, j\}}\left(I_{i k}+I_{j k}\right)
\end{gathered}
$$

With the arithmetic mean, we are able to compute the weights by using the reference subset $X_{R}=$ $\left\{a_{0}, a_{i}, \forall i \in N\right\}$ (see MACBETH methodology [2]). For the 2-additive Choquet integral model, these alternatives are not sufficient to compute interaction between criteria, hence the elaboration of $\mathcal{B}$ by adding the alternatives $a_{i j}$. The Equations (10) and (11) show that the binary actions are directly related to the parameters of the 2-additive Choquet integral model. Therefore a preferential information on $\mathcal{B}$ given by the DM permits to determine entirely all the parameters of the model.

As shown by the previous equations (9),(10), (11) and Lemma 1, it should be sufficient to get some preferential information from the DM only on binary actions. To entirely determine the 2 -additive capacity this information is expressed by the following relations:

- $P=\{(x, y) \quad \in \quad \mathcal{B} \times \mathcal{B} \quad:$ DM strictly prefers $x$ to $y\}$,
- $I=\{(x, y) \in \mathcal{B} \times \mathcal{B}:$ DM is indifferent between $x$ and $y$ \}.

The relation $P$ is irreflexive and asymmetric while $I$ is reflexive and symmetric.

Definition 3. The ordinal information on $\mathcal{B}$ is the structure $\{P, I\}$.

These two relations are completed by adding the relation $M$ which models the natural relations of monotonicity between binary actions coming from the monotonicity conditions $\mu(\{i\}) \geq 0$ and $\mu(\{i, j\}) \geq \mu(\{i\})$ for a capacity $\mu$. For $(x, y) \in$ $\left\{\left(a_{i}, a_{0}\right), i \in N\right\} \cup\left\{\left(a_{i j}, a_{i}\right), i, j \in N, i \neq j\right\}$,

$$
x M y \text { if } \operatorname{not}(x(P \cup I) y) .
$$

Example 1. Mary wants to buy a digital camera for her next trip. To do this, she consults a website where she finds six propositions based on three criteria: resolution of the camera (expressed in million of pixels), price (expressed in euros) and zoom (expressed by a real number)

$\begin{array}{lccc}\text { Cameras } & 1: \text { Resolution } & 2: \text { Price } & 3: \text { Zoom } \\ a: \text { Nikon } & 6 & 150 & 5 \\ b: \text { Sony } & 7 & 180 & 5 \\ c: \text { Panasonic } & 10 & 155 & 4 \\ d: \text { Casio } & 12 & 175 & 5 \\ e: \text { Olympus } & 10 & 160 & 3 \\ f: \text { Kodak } & 8 & 165 & 4\end{array}$

Using our notations, we have $N=\{1,2,3\}, X_{1}=$ $[6,12], X_{2}=[150,180], X_{3}=[3,5]$ and $X=X_{1} \times$ $X_{2} \times X_{3}$.

Based on these reference levels, the set of binary actions is $\mathcal{B}=\left\{a_{0}, a_{1}, a_{2}, a_{3}, a_{12}, a_{13}, a_{23}\right\}$, where for instance the alternative $a_{12}$ refers to a camera for which Mary is satisfied on resolution and price, but neutral on zoom. In order to make her choice, Mary gives also the following ordinal information: $I=\left\{\left(a_{12}, a_{3}\right)\right\}, P=\left\{\left(a_{13}, a_{1}\right),\left(a_{2}, a_{0}\right)\right\}$. Hence we have $M=\left\{\left(a_{1}, a_{0}\right),\left(a_{3}, a_{0}\right)\right.$, $\left.\left(a_{12}, a_{1}\right),\left(a_{12}, a_{2}\right),\left(a_{13}, a_{3}\right),\left(a_{23}, a_{2}\right),\left(a_{23}, a_{3}\right)\right\}$.

\subsection{The representation of ordinal information by the Choquet integral}

An ordinal information $\{P, I\}$ is said to be representable by a 2-additive Choquet integral if there exists a 2 -additive capacity $\mu$ such that:

1. $\forall x, y \in \mathcal{B}, x P y \Rightarrow C_{\mu}(U(x))>C_{\mu}(U(y))$

2. $\forall x, y \in \mathcal{B}, x I y \Rightarrow C_{\mu}(U(x))=C_{\mu}(U(y))$. 
A characterization of an ordinal information is given by Mayag et al. [13]. This result, presented below, is based on the following property called MOPI:

\section{Definition 4. [MOPI property]}

1. For a binary relation $\mathcal{R}$ on $\mathcal{B}$ and $x, y$ elements of $\mathcal{B},\left\{x_{1}, x_{2}, \cdots, x_{p}\right\} \subseteq \mathcal{B}$ is a path of $\mathcal{R}$ from $x$ to $y$ if $x=x_{1} \mathcal{R} x_{2} \mathcal{R} \cdots \mathcal{R} x_{p-1} \mathcal{R} x_{p}=y$. $A$ path of $\mathcal{R}$ from $x$ to $x$ is called a cycle of $\mathcal{R}$.

- We denote $x T C y$ if there exists a path of $(P \cup I \cup M)$ from $x$ to $y$.

- A path $\left\{x_{1}, x_{2}, \ldots, x_{p}\right\}$ of $(P \cup I \cup M)$ is said to be a strict path from $x$ to $y$ if there $e x$ ists $i$ in $\{1, \ldots, p-1\}$ such that $x_{i} P x_{i+1}$. In this case, we will write $x T C_{P} y$.

- We write $x \sim y$ if there exists a nonstrict cycle of $(P \cup I \cup M)$ (hence a cycle of $(I \cup$ $M)$ ) containing $x$ and $y$.

2. Let $i, j, k \in N$, $i$ fixed. We call Monotonicity of Preferential Information in $\{i, j, k\}$ w.r.t. $i$ the following property (denoted by $(\{i, j, k\}, i)$ $M O P I)$ :

$$
\begin{aligned}
& \left\{\begin{array}{l}
a_{i j} \sim a_{i} \\
a_{i k} \sim a_{k}
\end{array} \Rightarrow \operatorname{not}\left(a_{j} T C_{P} a_{0}\right)\right. \\
& \text { and } \\
& \left\{\begin{aligned}
a_{i j} & \sim a_{j} \\
a_{i k} & \sim a_{k}
\end{aligned} \Rightarrow \operatorname{not}\left(a_{i} T C_{P} a_{0}\right)\right. \\
& \left\{\begin{array}{l}
a_{i j} \sim a_{j} \\
a_{i k} \sim a_{i}
\end{array} \Rightarrow \operatorname{not}\left(a_{k} T C_{P} a_{0}\right) .\right.
\end{aligned}
$$

3. We say that, the set $\{i, j, k\}$ satisfies the property of MOnotonicity of Preferential Information (MOPI) if $\forall l \in\{i, j, k\},(\{i, j, k\}, l)$ MOPI is satisfied.

Theorem 1. An ordinal information $\{P, I\}$ is representable by a 2-additive Choquet integral on $\mathcal{B}$ if and only if the following two conditions are satisfied:

1. $(P \cup I \cup M)$ contains no strict cycle;

2. Any subset $K$ of $N$ such that $|K|=3$ satisfies the MOPI property.

Proof. See [13].

If there exists a strict cycle $\mathcal{C}$ contained in the relation $(P \cup I \cup M)$, we compute the set $P_{\mathcal{C}}=$ $\{(x, y) \in P$ such that $x \in \mathcal{C}$ and $y \in \mathcal{C}\}$ of all the strict preferences contained in $\mathcal{C}$. To solve this type of inconsistency, we propose to the DM, for all $(x, y) \in P_{\mathcal{C}}$, to change the preference $(x, y) \in P$ by the preference $(x, y) \in I$ or he can say nothing about the new preference between $x$ and $y$. In the next section, we treat the case of a violated MOPI condition.

\section{Dealing with inconsistencies coming from the violation of the MOPI property}

In this section, we suppose

1. $(P \cup I \cup M)$ contains no strict cycle;

2. The property MOPI is violated ,i.e., there exist $i, j, k \in N, l \in\{i, j, k\} \backslash\{i \vee k, i \vee j\}, i$ fixed such that:

$$
\left\{\begin{array}{l}
a_{i j} \sim a_{i \vee j} \\
a_{i k} \sim a_{i \vee k} \\
i \vee j \neq i \vee k
\end{array} \quad \text { and } \quad a_{l} T C_{P} a_{0}\right.
$$

For all $i, j$ in $N$, the element $i \vee j$ denotes one of the two elements $i, j$

According to the definition of MOPI property, we have only two ways to deal with inconsistencies in the DM's preferences:

(i) By restoring the relation $\operatorname{not}\left(a_{l} T C_{P} a_{0}\right)$;

(ii) By modifying the equivalence relation $\sim$ in $a_{i j} \sim a_{i \vee j}$ or $a_{i k} \sim a_{i \vee k}$.

\subsection{Restoration of $\operatorname{not}\left(a_{l} T C_{P} a_{0}\right)$}

\subsubsection{First approach: replacement of all pathologic strict relations $P$ by the indifference $I$}

We suggest to the DM to restore the relation $\operatorname{not}\left(a_{l} T C_{P} a_{0}\right)$ in two steps:

I- Step 1: Compute the set

$T C_{P}\left(a_{l}\right)=\left\{(x, y) \in P\right.$ such that $\left.a_{l} T C x P y\right\}$

The set $T C_{P}\left(a_{l}\right)$ is nonempty because we supposed $a_{l} T C_{P} a_{0}$.

II- Step 2: for each couple $(x, y)$ of $T C_{P}\left(a_{l}\right)$, follow one of these two recommendations:

(a) remove the relation $P$ between $x$ and $y$, and replace it by $I$; Hence we suggest him to say now $x I y$.

(b) remove the relation $P$ between $x$ and $y$, and do not replace it by any other relation $(x I y$ or $y P x)$. In this case, the new judgement of DM can be assimilated to "now, I am no more able to compare $x$ and $y "$.

\section{Remark 1.}

1. If the DM follows the recommendations IIa or $I I b$ then we have not $\left(a_{l} T C_{P} a_{0}\right)$.

2. Let us suppose that, before the recommendations IIa and IIb, there exist $r, s, t \in N$ such that not $\left(a_{r s} \sim a_{r \vee s}\right)$,i.e., there is no path from $a_{r \vee s}$ to $a_{r s}$. 


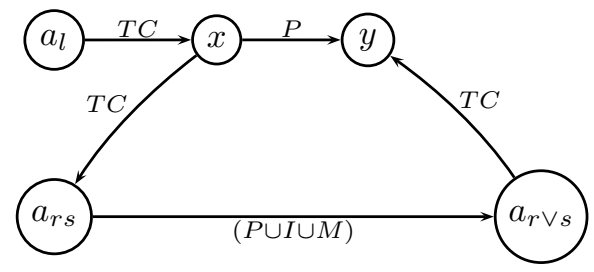

After the recommendations IIa and IIb

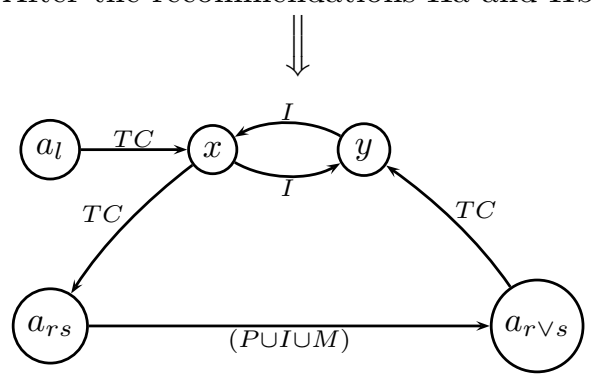

Figure 1: After the recommendations IIa and IIb, $a_{r s}$ and $a_{r \vee s}$ are now in a cycle

If after the recommendations IIa and IIb we have $a_{r s} \sim a_{r \vee s}$, then the existence of the new path $a_{r \vee s}$ to $a_{r s}$ is caused by an element of $(x, y)$ of $T C_{P}\left(a_{l}\right)$ for which the DM choose to replace $x P$ y by $x I y$ (see Figure 1). Hence, there exist two binary actions $x$ and $y$ such that:

- before the recommendations IIa and IIb, we had

$$
\left\{\begin{array}{l}
(x, y) \in T C_{P}\left(a_{l}\right) \\
x T C a_{r s} \\
a_{r \vee s} T C y
\end{array}\right.
$$

- after the recommendations IIa and IIb, we have now

$$
\left\{\begin{array}{l}
x I y \\
x T C a_{r s} \\
a_{r \vee s} T C y
\end{array}\right.
$$

The new cycle containing $a_{r s}$ and $a_{r \vee s}$ is nonstrict. If not, the exist $z, w \in \mathcal{B}$ such that $a_{l} T C x T C$ y TC $z P$ w TC $a_{0}$, a contradiction with not $\left(a_{l} T C_{P} a_{0}\right)$ obtained after the recommendations IIa and IIb.

The second point of the previous remark shows that, if the DM follows recommendations IIa and IIb then it is possible to have new nonstrict cycles in the relation $(P \cup I \cup M)$ and maybe the creation of new MOPI conditions. Therefore, in order to have efficient recommendations, we have to ensure that each MOPI condition created after the recommendations IIa and IIb is not violated. This is the purpose of the Proposition 1 based on the following definition:

Definition 5. Let $(x, y) \in T C_{P}\left(a_{l}\right)$.

We say that, the replacement of a $P$ by a $I$ between two binary actions $x$ and $y$ leads to the creation of the premises of a new MOPI condition, if there exist $r, s, t \in N, r$ fixed such that:
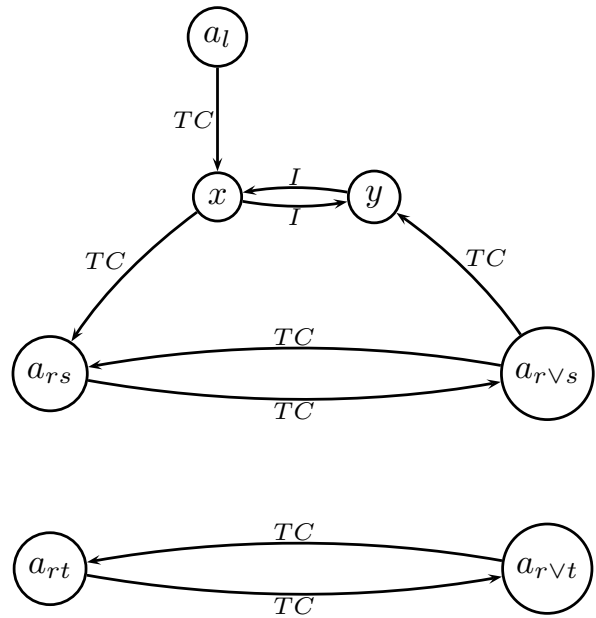

Figure 2: Configuration of the system given by the equation (15)

- before the recommendations IIa and IIb, we had:

$$
\operatorname{not}\left(a_{r s} \sim a_{r \vee s}\right) \quad \text { or } \quad \operatorname{not}\left(a_{r t} \sim a_{r \vee t}\right)
$$

- after the recommendations IIa and IIb, we have:

$$
\left\{\begin{array}{l}
a_{r s} \sim a_{r \vee s} \\
a_{r t} \sim a_{r \vee t} \\
r \vee s \neq r \vee t \\
x T C a_{r s} \\
x I y \\
a_{r \vee s} T C y
\end{array}\right.
$$

This system is illustrated by the Figure 2.

Proposition 1. Let $(x, y) \in T C_{P}\left(a_{l}\right)$.

If the replacement of a $P$ by a I between two binary actions $x$ and $y$ leads to the creation of the premises of a new MOPI condition, then this MOPI condition is necessarily satisfied.

Proof. Let us suppose that there exist $r, s, t \in N, r$ fixed such that:

- before the recommendations IIa and IIb, we had:

$$
\operatorname{not}\left(a_{r s} \sim a_{r \vee s}\right) \quad \text { or } \quad \operatorname{not}\left(a_{r t} \sim a_{r \vee t}\right)
$$

- after the recommendations IIa and IIb, we have:

$$
\left\{\begin{array}{l}
a_{r s} \sim a_{r \vee s} \\
a_{r t} \sim a_{r \vee t} \\
r \vee s \neq r \vee t \\
x T C a_{r s} \\
x I y \\
a_{r \vee s} T C y
\end{array}\right.
$$

We know that, after the recommendations IIa and IIb, we have now $\operatorname{not}\left(a_{l} T C_{P} a_{o}\right)$.

To show that the new MOPI condition created is satisfied, it is sufficient to prove $\operatorname{not}\left(a_{m} T C_{P} a_{0}\right)$, $m \in\{r, s, t\} \backslash\{r \vee s, r \vee t\}$. 
Let us suppose the contrary. Following the values of $m$, we have three cases:

1. The case $m=s$ :

$$
[m=s] \Rightarrow\left\{\begin{array}{l}
a_{r s} \sim a_{r} \\
a_{r t} \sim a_{t} \\
x T C a_{r s} \\
x I y \\
a_{s} T C_{P} a_{0}
\end{array}\right.
$$

Hence, we have $a_{l} T C x T C a_{r s} M a_{s} T C_{P} a_{0}$, i.e., $a_{l} T C_{P} a_{0}$, a contradition with the hypothesis $\operatorname{not}\left(a_{l} T C_{P} a_{o}\right)$.

\section{The case $m=r$ :}

$$
[m=r] \Rightarrow\left\{\begin{array}{l}
a_{r s} \sim a_{s} \\
a_{r t} \sim a_{t} \\
x T C a_{r s} \\
x I y \\
a_{r} T C_{P} a_{0}
\end{array}\right.
$$

Therefore $a_{l} \quad T C \quad x \quad T C \quad a_{r s} \quad M \quad a_{r} \quad T C_{P} a_{0}$,i.e., $a_{l} T C_{P} a_{0}$, a contradition with the hypothesis $\operatorname{not}\left(a_{l} T C_{P} a_{o}\right)$.

\section{The case $m=t$ :}

$$
[m=t] \Rightarrow\left\{\begin{array}{l}
a_{r s} \sim a_{s} \\
a_{r t} \sim a_{r} \\
x T C a_{r s} \\
x I y \\
a_{t} T C_{P} a_{0}
\end{array}\right.
$$

Hence we have $a_{l} T C x T C a_{r s} M a_{r} \sim$ $a_{r t} M a_{t} T C_{P} a_{0}$, i.e., $a_{l} T C_{P} a_{0}$, a contradiction with the hypothesis $\operatorname{not}\left(a_{l} T C_{P} a_{o}\right)$

We proved $\operatorname{not}\left(a_{m} T C_{P} a_{0}\right)$ for $m \in\{r, s, t\} \backslash\{r \vee$ $s, r \vee t\}$.

\subsubsection{Second approach: inversion of pathologic relations $P$}

To solve the inconsistencies coming from the violation of MOPI condition, the DM can also choose to change the preference $x P y$ by the preference $y P x$ where $(x, y) \in T C_{P}\left(a_{l}\right)$. But, the inversion of the relation $P$ does not guarantee that the new ordinal information is consistent as shown by the following Examples 2 and 3:

Example 2. $N=\{1,2,3,4\}, \quad I=$ $\left\{\left(a_{12}, a_{1}\right),\left(a_{13}, a_{3}\right),\left(a_{24}, a_{2}\right)\right\}$ and $P=\left\{\left(a_{2}, a_{4}\right)\right\}$.

It is obvious that there is no strict cycle in $(P \cup I \cup$ $M)$, but the MOPI condition is not satisfied because we have:

$$
\left\{\begin{array}{lll}
a_{12} & I & a_{1} \\
a_{13} & I & a_{3}
\end{array} \text { and } a_{2} P a_{4} M a_{0}\right.
$$

Changing the preference $a_{2} P a_{4}$ to $a_{4} P a_{2}$ leads to the satisfaction of this MOPI condition. But, if the DM chooses this recommendation then a strict cycle

$$
a_{4} P a_{2} I a_{24} M a_{4} .
$$

will be created.
Example 3. $N=\{1,2,3,4,5,6\}, \quad I=$ $\left\{\left(a_{12}, a_{1}\right),\left(a_{13}, a_{3}\right),\left(a_{45}, a_{5}\right),\left(a_{46}, a_{6}\right)\right\}$ and $P=$ $\left\{\left(a_{2}, a_{4}\right)\right\}$.

It is obvious that there is no strict cycle in $(P \cup I \cup$ $M)$, but the MOPI condition is not satisfied because we have:

$$
\left\{\begin{array}{lll}
a_{12} & I & a_{1} \\
a_{13} & I & a_{3}
\end{array} \text { and } a_{2} P a_{4} M a_{0} .\right.
$$

Changing the preference $a_{2} P a_{4}$ to $a_{4} P a_{2}$ leads to the satisfaction of this MOPI condition. But, if the DM chooses this recommendation then we will have a new violated MOPI condition:

$$
\left\{\begin{array}{lll}
a_{45} & I & a_{5} \\
a_{46} & I & a_{6}
\end{array} \text { and } a_{4} P a_{2} M a_{0} .\right.
$$

\subsection{Third approach: Modification of the equivalence relation $\sim$ in $a_{i j} \sim a_{i \vee j}$ or $a_{i k} \sim a_{i \vee k}$}

The objective of this recommendation is to "break" the relation $\sim$ between $a_{i j}$ and $a_{i \vee j}$ or between $a_{i k}$ and $a_{i \vee k}$. To do this, deleting all paths from $a_{i \vee j}$ to $a_{i j}$ or paths from $a_{i \vee k}$ to $a_{i k}$ is sufficient. Because the binary actions $a_{i j}$ and $a_{i \vee j}$ are contained in a nonstrict cycle, there exist on all these paths, two elements $x$ and $y$ such that $a_{i \vee j} T C$ y I x TC $a_{i \vee j}$. To restore the consistency of the MOPI condition, we can ask to the DM:

1. to replace $y I x$ by $x P y$ if $\operatorname{not}(y M x)$;

2. to declare that he is not able to compare now $x$ and $y$.

We propose him to do the same thing if he wants to delete paths from $a_{i \vee k}$ to $a_{i k}$.

Unfortunately, this solution is not satisfactory because it can lead to the creation of a violated MOPI condition as shown in the following example:

Example 4. $N=\{1,2,3,4,5,6\}, \quad I=$ $\left\{\left(a_{12}, a_{1}\right),\left(a_{13}, a_{4}\right),\left(a_{3}, a_{4}\right),\left(a_{45}, a_{5}\right),\left(a_{46}, a_{6}\right)\right\}$ and $P=\left\{\left(a_{2}, a_{0}\right)\right\}$.

It is easy to see that $(P \cup I \cup M)$ has no strict cycle, but the MOPI condition is violated because we have:

$$
\left\{\begin{array}{l}
a_{12} I a_{1} \\
a_{13} \sim a_{3}
\end{array} \quad \text { and } a_{2} P a_{0} .\right.
$$

If we follow the previous recommendations, in order to restore the consistency, the DM "break" the relation $\sim$ between $a_{13}$ and $a_{3}$ by replacing the relations $a_{13} \quad I \quad a_{4}$ by $a_{4} \quad P \quad a_{13}$ and $a_{3} I a_{4}$ by $a_{4} P a_{3}$. With these replacements, we get a new violated MOPI condition:

$$
\left\{\begin{array}{lll}
a_{45} & I & a_{5} \\
a_{46} & I & a_{6}
\end{array} \text { and } a_{4} P a_{3} M a_{0} .\right.
$$


The first of the three approaches of dealing with inconsistencies when the MOPI property is violated seems to be the most interesting. The recommendations proposed by this approach to the DM do not create new inconsistencies, apart those already existing. The number of inconsistencies is therefore reduced gradually as the DM provides consistent judgements with this approach. It is the main advantage compared to the other approaches which do not guarantee the decrease of inconsistencies. Therefore, we proposed below an algorithm to deal with inconsistencies in the representation of an ordinal information by a 2 -additive Choquet integral.

\section{Algorithm}

Let $G$ an oriented graph. Let be $S C C(G)$ the routine which builds the set of all strongly connected components of $G$. Let us consider the set NBA (Neutral Binary Actions) initialized at $\emptyset$.

Input: $N, \mathcal{B}, P, I$

Output: A consistent ordinal information $\{P, I\}$.

1. Complete the relations $\{P, I\}$ by adding the relation $M$ :

$$
M=\left\{\left(a_{i}, a_{0}\right) ;\left(a_{i j}, a_{i}\right), i, j \in N, i \neq j\right\} \backslash(P \cup I)
$$

2. Build the oriented graph $G=(V, E)$ where

- $V:=\mathcal{B}$ is the set of vertices of $G$

- $E: P \cup I \cup M$ is the set of edges

3. Compute the set $S C C(G)$;

\section{First test of inconsistencies:}

For $A \in S C C(G)$,

- If there exist $a, b \in A$ such that $(a, b) \in P$ return FALSE; (the preferential information $\{P, I\}$ given by the DM contains a strict cycle formed by the elements of $A$.) Recommendations to the DM:

If two elements $a$ and $b$ of $A$ form a couple $(a, b)$ of $P$, we propose to the DM to replace $(a, b) \in P$ by $(a, b) \in I$. He may choose also to make no other judgement between $a$ and $b$.

- Else go to Step 5;

\section{Second test of inconsistencies:}

For $i, j, k$ in $N$ such that $i$ is fixed, do:

If $\left\{\begin{array}{c}a_{i j} \text { and } a_{i \vee j} \text { are in the same } \\ \text { strong conected component, } \\ \text { and } \\ a_{i k} \text { and } a_{i \vee k} \text { are in the same } \\ \text { strong conected component, }\end{array}\right.$

- If there is no strict path from $a_{l}(l \in$ $\{i, j, k\} \backslash\{i \vee j, i \vee k\})$ to $a_{0}$, then add $a_{l}$ in NBA.
- Else return FALSE (the MOPI condition is violated).

\section{Recommendations to the DM:}

(a) Identify all vertices $x$ and $y$ for which $(x, y) \in P$ and such that there exists a path from $a_{l}$ to $x$;

(b) For each couple $(x, y)$ identified, we propose to the DM to replace $(x, y) \in$ $P$ by $(x, y) \in I$. He may choose also to make no other judgement between $x$ and $y$.

6. The ordinal information $\{P, I\}$ is consistent.

This algorithm was implemented in JAVA language in a THALES's software called MYRIAD. For more details about this implementation, see [14].

\section{References}

[1] S. Angilella, S. Greco, and B. Matarazzo. Nonadditive robust ordinal regression: A multiple criteria decision model based on the Choquet integral. European Journal of Operational Research, 41(1):277-288, 2009.

[2] C. A. Bana e Costa, J.-M. De Corte, and J.C. Vansnick. On the mathematical foundations of MACBETH. In J. Figueira, S. Greco, and M. Ehrgott, editors, Multiple Criteria Decision Analysis: State of the Art Surveys, pages 409437. Springer, 2005.

[3] A. Chateauneuf and J.Y. Jaffray. Some characterizations of lower probabilities and other monotone capacities through the use of Möbius inversion. Mathematical Social Sciences, 17:263-283, 1989.

[4] V. Clivillé, L. Berrah, and G. Mauris. Quantitative expression and aggregation of performance measurements based on the MACBETH multi-criteria method. International Journal of Production economics, 105:171-189, 2007.

[5] J. R. Figueira, S. Greco, and R. Slowinski. Building a set of additive value functions representing a reference preorder and intensities of preference : Grip method. European Journal of Operational Research, 195(2):460-486, 2009.

[6] M. Grabisch. $k$-order additive discrete fuzzy measures and their representation. Fuzzy Sets and Systems, 92:167-189, 1997.

[7] M. Grabisch. The Möbius transform on symmetric ordered structures and its application to capacities on finite sets. Discrete Mathematics, 287 (1-3):17-34, 2004.

[8] M. Grabisch and C. Labreuche. Fuzzy measures and integrals in MCDA. In J. Figueira, S. Greco, and M. Ehrgott, editors, Multiple Criteria Decision Analysis: State of the Art Surveys, pages 565-608. Springer, 2005.

[9] M. Grabisch and Ch. Labreuche. A decade of application of the Choquet and Sugeno inte- 
grals in multi-criteria decision aid. $4 O R, 6: 1-$ 44, 2008.

[10] M. Grabisch, Ch. Labreuche, and J.-C. Vansnick. On the extension of pseudo-Boolean functions for the aggregation of interacting bipolar criteria. Eur. J. of Operational Research, 148:28-47, 2003.

[11] S. Greco, V. Mousseau, and R. Slowinski. Ordinal regression revisited: Multiple criteria ranking using a set of additive value functions. European Journal of Operational Research, 51(2):416-436, 2008.

[12] Ch. Labreuche and M. Grabisch. The Choquet integral for the aggregation of interval scales in multicriteria decision making. Fuzzy Sets and Systems, 137:11-26, 2003.

[13] B. Mayag, M. Grabisch, and C. Labreuche. A representation of preferences by the Choquet integral with respect to a 2 -additive capacity. Theory and Decision, DOI: 10.1007/s11238010-9198-3, forthcoming. Available at http://www.springerlink.com/content/313t22t08v722h82/.

[14] Brice Mayag. Elaboration d'une démarche constructive prenant en compte les interactions entre critères en aide multicritère à la décision. $\mathrm{PhD}$ thesis, University of Paris 1 Panthéon-Sorbonne, Paris, 2010. Available at http://sites.google.com/site/bricemayag/aboutmy-phd.

[15] T. Murofushi and S. Soneda. Techniques for reading fuzzy measures (III): interaction index. In 9 th Fuzzy System Symposium, pages 693-696, Sapporo, Japan, May 1993. In Japanese.

[16] J.P. Pignon and Ch. Labreuche. A methodological approach for operational and technical experimentation based evaluation of systems of systems architectures. In Int. Conference on Software 8 Systems Engineering and their Applications (ICSSEA), Paris, France, December 4-6 2007.

[17] L. S. Shapley. A value for $n$-person games. In H. W. Kuhn and A. W. Tucker, editors, Contributions to the Theory of Games, Vol. II, number 28 in Annals of Mathematics Studies, pages 307-317. Princeton University Press, 1953.

[18] H. Simon. Rational choice and the structure of the environment. Psychological Review, 63(2):129-138, 1956.

[19] Y. Siskos, E. Grigoroudis, and N. F. Matsatsinis. Uta methods. In J. Figueira, S. Greco, and M. Ehrgott, editors, Multiple Criteria Decision Analysis: State of the Art Surveys, pages 297-343. Springer, 2005.

[20] P. Slovic, M. Finucane, E. Peters, and D. G. MacGregor. The affect heuristic. In T. Gilovitch, D. Griffin, and D. Kahneman, editors, Heuristics and biases: the psychology of intuitive judgment, pages 397-420. Cambridge University Press, 2002.

[21] A. Tversky and D. Kahneman. Advances in prospect theory: cumulative representation of uncertainty. J. of Risk and Uncertainty, 5:297-323, 1992. 